\title{
Linear evolution of sandwave packets
}

\author{
P. C. Roos, ${ }^{1}$ P. Blondeaux, ${ }^{2}$ S. J. M. H. Hulscher, ${ }^{1}$ and G. Vittori ${ }^{2}$ \\ Received 28 June 2004; revised 12 January 2005; accepted 17 February 2005; published 23 September 2005.
}

[1] We investigate how a local topographic disturbance of a flat seabed may become morphodynamically active, according to the linear instability mechanism which gives rise to sandwave formation. The seabed evolution follows from a Fourier integral, which can generally not be evaluated in closed form. As numerical integration is rather cumbersome and not transparent, we propose an analytical way to approximate the solution. This method, using properties of the fastest growing mode only, turns out to be quick, insightful, and to perform well. It shows how a local disturbance develops gradually into a sandwave packet, the area of which increases roughly linearly with time. The elevation at the packet's center ultimately tends to increase, but this may be preceded by an initial stage of decrease, depending on the spatial extent of the initial disturbance. In the case of tidal asymmetry, the individual sandwaves in the packet migrate at the migration speed of the fastest growing mode, whereas the envelope moves at the group speed. Finally, we apply the theory to trenches and pits and show where results differ from an earlier study in which sandwave dynamics have been ignored.

Citation: Roos, P. C., P. Blondeaux, S. J. M. H. Hulscher, and G. Vittori (2005), Linear evolution of sandwave packets, J. Geophys. Res., 110, F04S14, doi:10.1029/2004JF000196.

\section{Introduction}

[2] Tidal sandwaves form a prominent bed feature in tidedominated offshore areas of shallow shelf seas. They occur in rhythmic patches, with crests spaced several hundreds of meters apart (Figure 1), several meters high and oriented almost perpendicularly to the principal direction of the tidal flow [Terwindt, 1971].

[3] Sandwaves are highly dynamic, with typical timescales of formation of the order of years and migration speeds up to $10 \mathrm{~m} \mathrm{yr}^{-1}$. Insight in sandwave dynamics, particularly their migration, has several practical applications, as it may help optimizing dredging strategies, and ascertaining pipeline safety [Németh et al., 2003].

[4] Using a process-based morphodynamic model, Hulscher [1996] showed that the formation of tidal sandwaves can be explained as a linear instability of a flat seabed, driven by the vertical flow circulations induced by tide-topography interactions. This theory was later extended and refined with respect to the description of hydrodynamics and solution procedure [Gerkema, 2000; Komarova and Hulscher, 2000; Besio et al., 2003a]. Of practical interest is the modeling of sandwave migration by including a residual current [Németh et al., 2002] and tidal asymmetry [Besio et al., 2003b; Besio et al., 2004]. In the studies cited here the main focus is on the so-called fastest growing mode (fgm), that is, on the wavelength for which the growth rate attains

\footnotetext{
${ }^{1}$ Water Engineering and Management, Faculty of Engineering Technology, University of Twente, Enschede, Netherlands.

${ }^{2}$ Department of Environmental Engineering, University of Genova, Genova, Italy.
}

Copyright 2005 by the American Geophysical Union. 0148-0227/05/2004JF000196\$09.00 its maximum. The models therefore essentially describe rhythmic sandwave patterns of infinite spatial extent.

[5] In the present study, however, we focus on the evolution of a local, or isolated topographic disturbance on an otherwise flat seabed. How will such a disturbance evolve in time, according to the hydrodynamic and morphodynamic mechanisms of sandwave formation?

[6] We follow an approach which fully relies on the linear theories cited above. We particularly use the dispersion relationship for sandwaves, that is, the relation between growth rate (and migration rate) of sandwaves and the topographic wave numbers. The study of a spatially constrained topography, however, involves the dynamics of the full spectrum rather than that of the fastest growing mode only. Essential is that we use the general properties of the dispersion relationship, so we do not need to revisit the details of the underlying hydrodynamics and sediment transport. The solution to the problem, that is, the bed topography as a function of both space and time, follows from a Fourier integral which can generally not be evaluated in closed form. We explore and compare two solution methods: numerical integration and an analytical approximation of the dispersion relation. The former method is direct and accurate, but not transparent and computationally expensive, especially in three dimensions. The approximate method, based on an asymptotic Gaussian model used previously in a hydrodynamic context [Benjamin, 1961; Gaster, 1968; Gaster and Davey, 2003; Gaster, 1981, 1982], combines a quadratic approximation of the dispersion relation (around the fgm) with suitable initial conditions. This Gaussian model is also known as Method of Steepest Descent or Saddle-Point Method [Morse and Feshbach, 1953]. The analytical solution, while giving insight in the underlying physics, turns out to be quick 


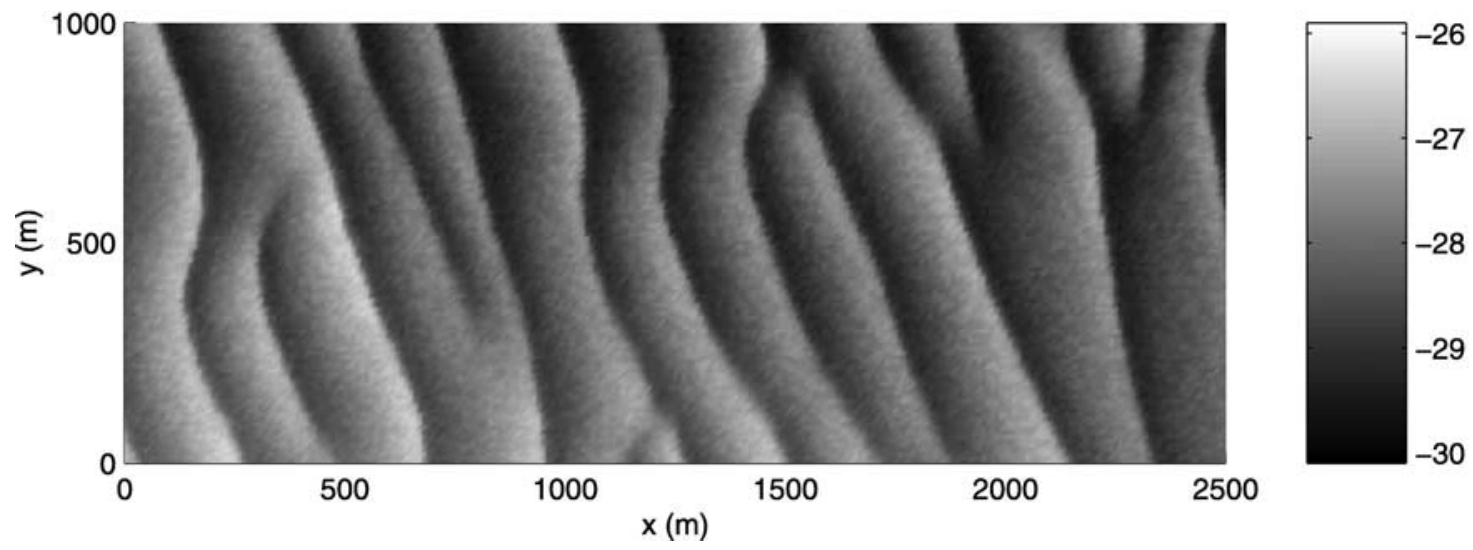

Figure 1. Sandwave field in the North Sea (bed level in meters, data from North Sea Directorate, The Netherlands).

and to perform well. To obtain the input parameters of this model, we use the three-dimensional extension, neglecting Coriolis effects, of the code of Besio et al. [2003a] (hereinafter referred to as $\mathrm{BBF}$ ) (for a description of this model, see Appendix B).

[7] Finally, we apply the theory to the practical case of a dredged channel or a sandpit, focusing on length scales of sandwaves (several hundreds of meters). What is the influence of such a human intervention on the short and intermediate term morphodynamics of the seabed? We compare our results with those from a different model [van de Kreeke et al., 2002], in which a depth-averaged flow approach has been adopted, neglecting the details of the vertical flow structure and, hence, neglecting the dynamics of sandwaves.

[8] This paper is organized as follows. Section 2 provides a general background on sandwave dynamics. In section 3 we present our analysis of the sandwave packets and a description of the numerical method. In section 4 , we compare the analytical approximation with the numerical method and we apply the analysis to study the evolution of a dredged trench. Finally, sections 5 and 6 contain the discussion and conclusions, respectively.

\section{Theoretical Background}

[9] Consider a coastal region of uniform depth $H^{*}$ (dimensional quantities are denoted with an asterisk). Let $x^{*}$ and $y^{*}$ denote the horizontal coordinates parallel and perpendicular to the direction of the tidal flow, which now is supposed to be unidirectional. The $z^{*}$ axis points upward with the location of the seabed at $z^{*}=z_{b}^{*}\left(x^{*}, y^{*}, t^{*}\right)$. If a three-dimensional bottom perturbation of small amplitude is considered, a linear stability analysis like the one by Hulscher [1996] (or the later extensions, see section 1) provides the complex amplification rate $\omega^{*}=\omega_{r}^{*}+i \omega_{i}^{*}$ as a function of the topographic wave numbers $k^{*}$ and $\ell^{*}$. This is the dispersion relationship for sandwaves, essentially containing all the properties of sandwave dynamics that are described by linear theory. We highlight the following properties.

[10] It is sufficient to define $\omega^{*}$ for $k^{*} \geq 0$, as the perturbations with wave vectors $\left(k^{*}, \ell^{*}\right)$ and $-\left(k^{*}, \ell^{*}\right)$ have complex conjugate growth rates. Furthermore, at the length scales of sandwaves, Coriolis effects have a negligible effect, which causes $\omega^{*}$ to be symmetric in $\ell^{*}$. In particular, a value $\left(\hat{k}^{*}, 0\right)$ can be singled out such that $\omega^{*}{ }_{r}$ is maximum: the fastest growing mode (fgm). The symmetry in $\ell^{*}$ causes the fgm to be attained at $\ell^{*}=$ 0 , that is, for a crest orientation perpendicular to the tidal flow. This explains why many studies have been restricted to topographic variations in the $x^{*}$ direction only, effectively taking $\ell^{*}=0$ [Gerkema, 2000; Komarova and Hulscher, 2000; Németh et al., 2002; Besio et al., 2003a, $2003 \mathrm{~b}$. For large wave numbers, $\omega_{r}^{*}$ decays roughly proportional to $-\left(k^{*^{2}}+\ell^{*^{2}}\right)$, due to the diffusive bed slope effects on sediment transport. This leads to a bounded region in the $\left(k^{*}, \ell^{*}\right)$ plane around the fgm where growth rates are positive. The imaginary part is related to the migration of the bottom forms, the corresponding migration speed being given by $-\omega_{i}^{*} /\left(k^{*^{2}}+\right.$ $\left.\ell^{* 2}\right)^{1 / 2}$. Migration only occurs when the symmetry of the $M_{2}$ tide is disrupted, for example by the presence of either a residual current [Németh et al., 2002], a superimposed $M_{4}$ component or both [Besio et al., 2003b]. Finally, conservation of sediment forces $\omega^{*}(0,0)=0$.

[11] Typical scales of the morphodynamic problem are horizontal length $2 \pi / \hat{k}^{*}(\sim 100-1000 \mathrm{~m}$ [e.g., see Hulscher, 1996; Besio et al., 2003b], vertical length given by the water depth $H^{*}(\sim 20-30 \mathrm{~m})$, time $1 / \hat{\omega}_{r}^{*}(\sim 1$ year $)$. So, let us define the following dimensionless quantities: $x=\hat{k}^{*} x^{*}, y=\hat{k}^{*} y^{*}, z=z^{*} / H^{*}, z_{b}=z_{b}^{*} / H^{*}, \tau=\hat{\omega}_{r}^{*} t^{*}$. As a result of this scaling, the maximum growth equals $\omega_{r}=1$, and is attained at $(k, \ell)=(1,0)$, as shown by Figure 2.

[12] According to the linear theory, the elevation of the seabed at time $\tau$ is given by

$$
z_{b}(x, y, \tau)=\int_{-\infty}^{\infty} \int_{0}^{\infty} \tilde{z}_{b}(k, \ell) e^{\omega(k, \ell) \tau} e^{i(k x+\ell y)} \mathrm{d} k \mathrm{~d} \ell+\text { c.c. }
$$

Here $\tilde{z}_{b}$ is the Fourier spectrum of the topography at $\tau=0$ and $\omega(k, \ell)$ is the dispersion relationship introduced above, now in dimensionless form.

[13] The evaluation of integral (1) depends not only on the form of the dispersion relationship but also on the initial topography. To allow for an analytical solution, we 
(a) real part

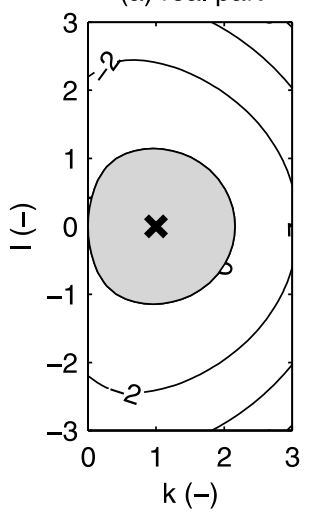

(b) imaginary part

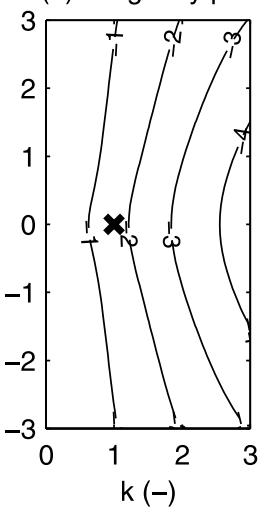

(c) migration rate

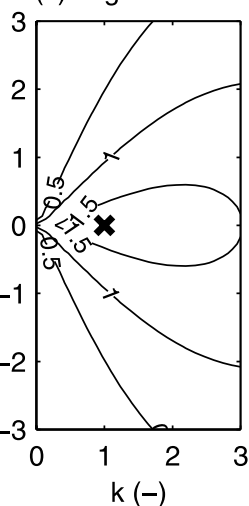

Figure 2. Example of the dispersion relation for sandwaves in dimensionless form: contour plots of (a) real part $\omega_{r}$ with positive values shaded, (b) imaginary part $\omega_{i}$, and (c) migration rate $-\omega_{i} /\left(k^{2}+\ell^{2}\right)^{1 / 2}$. The fastest growing mode $(\mathrm{fgm})(k, \ell)=(1,0)$ is denoted with a cross. Hydrodynamic conditions are taken from case II in Table 1.

restrict our attention to Gaussian initial topographies of the form

$$
\begin{aligned}
z_{b}(x, y, 0) & =\frac{\epsilon e^{-\left(x^{2} / L^{2}+y^{2} / B^{2}\right)}}{L B \pi} \\
& =\frac{\epsilon}{4 \pi^{2}} \int_{-\infty}^{\infty} \int_{-\infty}^{\infty} e^{-\frac{1}{4}\left(k^{2} L^{2}+\ell^{2} B^{2}\right)} e^{i(k x+\ell y)} \mathrm{d} k \mathrm{~d} \ell .
\end{aligned}
$$

This defines a type of topographic hump, $L$ and $B$ being its characteristic length and width in $x$ and $y$ direction, respectively. This topography has been defined such that $\iint z_{b} \mathrm{~d} x \mathrm{~d} y=\epsilon$, so $\epsilon$ is the amount of sand contained by the hump. Following the assumption of linearity, the maximum bed elevation $\epsilon(L B \pi)^{-1}$ should be small. For $L \downarrow 0$ and $B \downarrow 0$ the spectrum becomes uniform and the Gaussian shape reduces to a Dirac hump $\epsilon \delta(x) \delta(y)$. The quantity $\epsilon$ can be chosen negative to make equation (2) represent a trench or pit rather than a hump. The theory is linear in $\epsilon$, so the results will be identical except for a sign change. From equation (2), note that the extent of the spectrum in Fourier space $\left(\propto L^{-1}\right)$ and the extent of the topography in physical space $(\propto L)$ are inversely proportional (the same applies to $B$ ).

\section{Solution Method and Properties of the Solution}

\subsection{Introduction}

[14] We apply two methods to evaluate integral (1). First, in section 3.2, we propose an analytical method based on a classical Gaussian model, related to the Method of Steepest Descent or the Saddle-Point Method [Morse and Feshbach, 1953]. It has been successful in the context of boundary layers over a flat plate, that is, in describing the evolution of a point disturbance in such a system [Benjamin, 1961; Gaster, 1968; Gaster and Davey, 2003; Gaster, 1981, 1982], where the aim was to provide asymptotic approximations, valid for large values of $x, y$ and $\tau$. In the context of linear sandwave dynamics this may seem a bit awkward: when the asymptotic approximations are valid, the growing sandwaves have reached amplitudes that are likely to be beyond the applicability of the linear model (also see the discussion in section 5.2). Therefore we look for solutions which perform well also on the short and intermediate term. As our interest is furthermore on practical applications involving details of the initial geometry, we explicitly focus on the role of the geometrical characteristics $L$ and $B$. Finally, in section 3.2, we present the details of the alternative, numerical method.

\subsection{Gaussian Model}

[15] In the Gaussian model, the actual sandwave dispersion relation is approximated by a second-order polynomial around the fgm according to

$$
\omega^{\mathrm{app}}(k, \ell)=1-\lambda(k-1)^{2}-\mu \ell^{2}-i\left[\sigma+\sigma_{g}(k-1)\right] .
$$

Here we have introduced four parameters, which can be estimated from existing sandwave models; we use the BBF code (background in Appendix B). All parameters refer to the properties of the dispersion relationship in the fgm:

$$
\lambda=-\frac{1}{2} \frac{\widehat{\partial^{2} \omega}}{\partial k^{2}}, \quad \mu=-\frac{1}{2} \frac{\widehat{\partial^{2} \omega}}{\partial \ell^{2}}, \quad \sigma=-\hat{\omega}_{i}, \quad \sigma_{g}=-\frac{\widehat{\partial \omega_{i}}}{\partial k},
$$

where evaluation at $(k, \ell)=(1,0)$ is denoted with a hat. The parameters $\lambda=\lambda_{r}+i \lambda_{i}$ and $\mu=\mu_{r}+i \mu_{i}$ are generally complex, with positive real parts due to the fgm being a maximum. The real-valued parameters $\sigma$ and $\sigma_{g}$ are the migration speed and the group speed of the fgm, respectively. By the symmetry in $\ell$, the approximation (3) does not contain any terms in $\ell$ and $k \ell$. Note that the last property listed in section $2, \omega(0,0)=0$, is generally not satisfied. This concerns a single point, however, and the integrated result still satisfies conservation of sediment. The validity of this quadratic approximation will be further investigated in section 4.2.

[16] Now, the simple forms of both the initial topography (2) and the approximated dispersion relationship (3) allow us to evaluate the integral (1) in closed form. The solution can be written as

$$
\begin{aligned}
z_{b}^{\mathrm{app}}(x, y, \tau)= & \epsilon A_{L B}(\tau) E_{L B}(x, y, \tau) Q_{L}(x, \tau) \\
& \times e^{i\left\{\left[1-\gamma_{L}(\tau)\right] x-\left[\sigma-\sigma_{g} \gamma_{L}(\tau)\right] \tau\right\}}+\text { c.c. },
\end{aligned}
$$

where

$$
\begin{gathered}
A_{L B}(\tau)=\frac{e^{\left[1-\gamma_{L}(\tau)\right] \tau}}{\pi \sqrt{\left(L^{2}+4 \lambda \tau\right)\left(B^{2}+4 \mu \tau\right)}}, \\
E_{L B}(x, y, \tau)=\exp \left\{-\left(\frac{\left[x-\sigma_{g} \tau\right]^{2}}{L^{2}+4 \lambda \tau}+\frac{y^{2}}{B^{2}+4 \mu \tau}\right)\right\}, \\
Q_{L}(x, \tau)=\frac{1}{2}+\frac{1}{2} \operatorname{erf}\left\{\frac{2 \lambda \tau+i\left(x-\sigma_{g} \tau\right)}{\sqrt{L^{2}+4 \lambda \tau}}\right\}, \\
\gamma_{L}(\tau)=\frac{L^{2}}{L^{2}+4 \lambda \tau} .
\end{gathered}
$$


(a) envelope
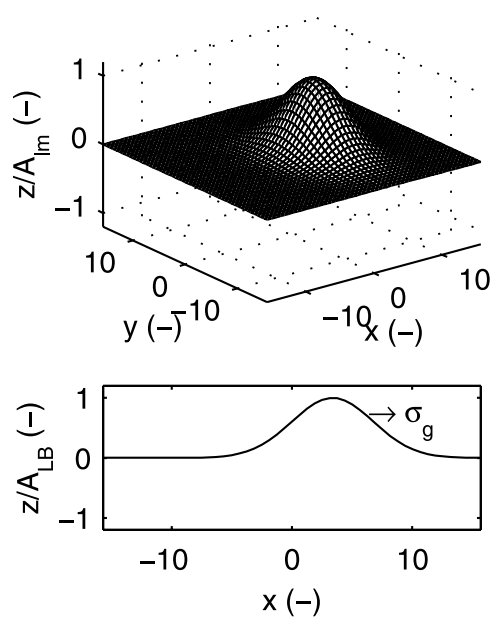

(b) pattern
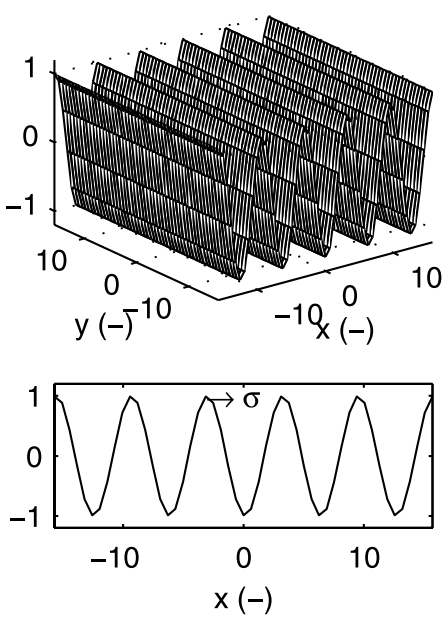

(c) envelope $\times$ pattern
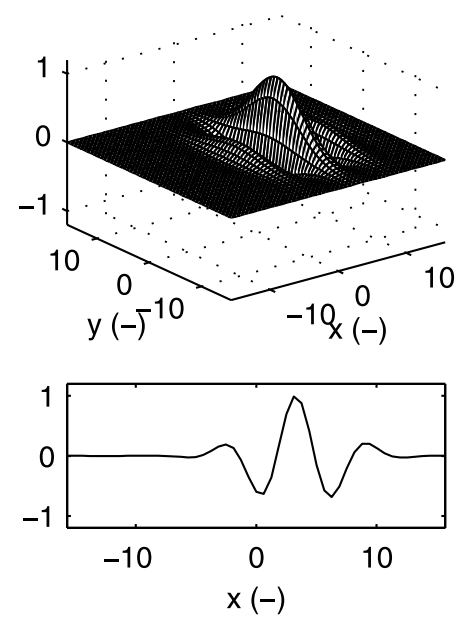

Figure 3. Sketch of the components of the analytical solution: (a) envelope $E_{L B}$ migrating at group velocity $\sigma_{g}$, (b) pattern migrating at phase speed $\sigma$, and (c) pattern with envelope. The top row shows three-dimensional plots, and the bottom row shows the values along $y=0$. We show the evolution at $\tau=$ 2, for $L=4$ and $B=6$, with the hydrodynamic conditions taken from case II in Table 1 .

The complex-valued factor $Q_{L}$, due to the lower integration bound at $k=0$ in (1), expresses a difference from the earlier use of this Gaussian model (see section 1).

[17] Now let us attempt to interpret the solution given by equations $(5)-(9)$. After noting that $\gamma_{0}(\tau)=0$, $\lim _{\tau \rightarrow \infty} \gamma_{L}(\tau)=0, \lim _{\tau \rightarrow \infty} \gamma_{L}(\tau) \tau=L^{2} /(4 \lambda)$ and that $\lim _{\tau \rightarrow \infty} Q_{L}=1$, we can identify some essential properties of the solution (5) at large times. Here, we will furthermore assume that both $\lambda$ and $\mu$ are real-valued, such that $A_{L B}$ and $E_{L B}$ are real-valued, as well.

[18] 1. A migrating sandwave packet emerges, consisting of a spatially periodic pattern with an envelope $E_{L B}$ (Figure 3 ). For large $\tau$, the harmonic part of equation (5) becomes $e^{i(x-\sigma \tau)}$, that is, the pattern has the characteristic wavelength and migration rate $\sigma$ of the fgm. The envelope, however, migrates at the group velocity $\sigma_{g}$, which may generally differ from $\sigma$. The center of the packet is therefore found at $(x, y)=\left(\sigma_{g} \tau, 0\right)$.

[19] 2. The bed elevation at the packet's center is given by the real-valued quantity $A_{L B}(\tau) Q_{L}\left(\sigma_{g} \tau, \tau\right)$ (real-valued, when evaluated at $x=\sigma_{g} \tau$ and for real-valued $\lambda$ ), which can be interpreted as a factor of amplification. The instability of the bed gives it a tendency to grow exponentially, but initially it may decay when the characteristic length $L$ and width $B$ are sufficiently small (Figure $4 a$ ). The gravitational bed slope effects on sediment transport then dominate the initial dynamics, lowering and widening the topographic hump.

[20] 3. The envelope $E_{L B}$ is constant along migrating and expanding ellipses, the area of which roughly expands linearly with time:

$$
\frac{\left[x-\sigma_{g} \tau\right]^{2}}{L^{2}+4 \lambda \tau}+\frac{y^{2}}{B^{2}+4 \mu \tau}=C^{2}
$$

with $C$ constant. The values of $L, B, \lambda$ and $\mu$ determine the shape of the ellipse; for large $\tau$ the axes ratio becomes $\lambda / \mu$. The envelope contour, which initially crosses the axis at
$x_{c}=L$ and $y_{c}=B$ (that is, for $C=1$ ), initially expands longitudinally and laterally according to

$$
\left.\frac{\mathrm{d} x_{c}}{\mathrm{~d} \tau}\right|_{\tau=0}=\left.\frac{2 \lambda}{L} \quad \frac{\mathrm{d} y_{c}}{\mathrm{~d} \tau}\right|_{\tau=0}=\frac{2 \mu}{B},
$$

respectively.

[21] The description of the expanding ellipses will be slightly distorted when accounting for the role of the complex-valued factor $Q_{L}$, which affects both amplitude/ envelope and phase of the packet. Moreover, considering complex-valued rather than real-valued $\lambda$ and $\mu$ affects the dynamics of the packet in the following way. The imaginary part $\lambda_{i}$ determines the variations of wavelength within the packet: if $\lambda_{i}<0$, the wavelength increases in the direction of migration, if $\lambda_{i}>0$ the opposite is true. The imaginary part $\mu_{i}$ determines the shape of individual sandwave crests: as $\mu_{i}<$ 0 generally holds, they are deformed into a barchan type of shape with its crest ends pointing in the direction of migration. (a) three dimensions

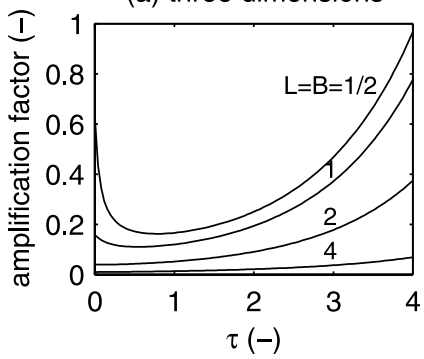

(b) two dimensions

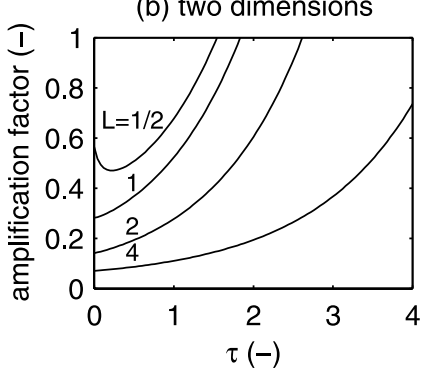

Figure 4. Time evolution of amplification factor (a) in three dimensions $\left(A_{L B}(\tau) Q_{L}\left(\sigma_{g} \tau, \tau\right)\right)$ and (b) in two dimensions $\left(A_{L}(\tau) Q_{L}\left(\sigma_{g} \tau, \tau\right)\right)$. Their evolution has been plotted for different values of $L(=B$, as chosen here); see labels. Parameter values are $\lambda=0.85$ and $\mu=1.26$ (case I in Table 1). 
Table 1. Overview of Tidal Conditions I-IV, for Each Case Showing the Characteristics of the Fastest Growing Mode (fgm) and Values of the Dimensionless Parameters in Equation (3) ${ }^{\mathrm{a}}$

\begin{tabular}{|c|c|c|c|c|c|}
\hline Symbol & Description & I & II & III & $\mathrm{IV}^{\mathrm{b}}$ \\
\hline$U_{M_{0}}^{*}$ & residual component, $\mathrm{m} \mathrm{s}^{-1}$ & 0.00 & 0.03 & 0.00 & 0.02 \\
\hline$U_{M_{2}}^{N_{0}}$ & amplitude of $M_{2}$ component, $\mathrm{m} \mathrm{s}^{-1}$ & 0.60 & 0.60 & 0.60 & 0.55 \\
\hline$U_{M_{4}}^{*_{2}}$ & amplitude if $M_{4}$ component, $\mathrm{m} \mathrm{s}^{-1}$ & 0.00 & 0.00 & 0.03 & 0.04 \\
\hline$\varphi_{M_{4}}$ & phase between peaks of $M_{4}$ and $M_{2}$, deg & - & - & 0 & 0 \\
\hline$H^{*}$ & mean water depth, $\mathrm{m}$ & 20 & 20 & 20 & 17 \\
\hline $2 \pi / \hat{k}^{*}$ & wavelength of fgm, $10^{2} \mathrm{~m}$ & 4.3 & 4.3 & 4.3 & 4.2 \\
\hline $1 / \hat{\omega}_{r}^{*}$ & e-folding time of growth for fgm, years & 10.9 & 10.8 & 10.9 & 11.7 \\
\hline$\sigma$ & migration speed of fgm & 0.00 & 1.64 & 0.33 & 1.69 \\
\hline$\sigma_{g}$ & fgm's group speed, envelope's migration speed & 0.00 & 1.69 & 0.34 & 1.74 \\
\hline$\lambda_{r}^{8}$ & curvature coefficient, determining longitudinal expansion of the packet & 0.85 & 0.85 & 0.85 & 0.89 \\
\hline$\lambda_{i}$ & curvature coefficient, determining wavelength variations within packet & 0.00 & -0.05 & -0.01 & -0.035 \\
\hline$\mu_{r}$ & curvature coefficient, determining lateral expansion of the packet & 1.26 & 1.26 & 1.26 & 1.27 \\
\hline$\underline{\mu_{i}}$ & curvature coefficient, determining the shape of the sandwave ends & 0.00 & -0.59 & -0.10 & -0.57 \\
\hline
\end{tabular}

Finally, we note that changing from three to two dimensions slightly affects the details of the analysis; expressions are given in Appendix A. For example, unlike the threedimensional result $A_{L B} \propto \tau^{-1} e^{\tau}$, in two dimensions we find $A_{L} \propto \tau^{-1 / 2} e^{\tau}$ (compare Figures $4 \mathrm{a}$ and $4 \mathrm{~b}$ ).

[22] A physical interpretation of the properties of the sandwave packet will be given in section 5.1.

\subsection{Numerical Integration}

[23] More directly, the integral (1) can be evaluated numerically, using the dispersion relation on a discrete grid $\left(k_{j}, \ell_{k}\right)$ with $k_{j}=j \Delta_{k}\left(j=0, \ldots, N_{k}\right)$ and $\ell_{k}=k \Delta_{\ell}\left(k=-N_{\ell}, \ldots\right.$, $\left.\ldots, N_{\ell}\right)$. This grid should cover all relevant modes, and the spacings $\Delta_{k}$ and $\Delta_{\ell}$ should be sufficiently small, as the grid inevitably introduces a periodic recurrence of the packet over the distances $2 \pi / \Delta_{k}$ and $2 \pi / \Delta_{\ell}$ in $x$ and $y$ direction, respectively. The initial topographic spectrum $Z_{j k}=\tilde{z}_{b}\left(k_{j}, \ell_{k}\right)$ and the dispersion relationship $\omega_{j k}=\omega\left(k_{j}, \ell_{k}\right)$ are known complex numbers, obtained from equation (2) and the BBF code, respectively. The numerical solution is then given by

$$
z_{b}^{\text {num }}(x, y, \tau)=\left[\Delta_{k} \Delta_{\ell} \sum_{j=1}^{N_{k}} \sum_{k=-N_{\ell}}^{N_{\ell}} \bar{Z}_{j k} e^{\bar{\omega}_{j k} \tau} e^{i\left(\bar{k}_{j} x+\ell_{k} y\right)}\right]+\text { c.c. }
$$

in which $\bar{Z}_{j k}=\frac{1}{2}\left(Z_{j-1, k}+Z_{j k}\right), \bar{\omega}_{j k}=\frac{1}{2}\left(\omega_{j-1, k}+\omega_{j k}\right)$ and $\bar{k}_{j}=\frac{1}{2}\left(k_{j-1}\right.$ $\frac{1}{2}\left(k_{j-1}+k_{j}\right)$. In the two-dimensional case, the numerical integration is done analogously, but neglecting the summation over the transverse wave number $\ell$; see Appendix A.

\section{Results}

\subsection{Hydrodynamic Conditions}

[24] In this section we compare the results of the numerical method with those of the analytical approximation. The properties of the latter have already been described in section 3.2. In the following, we distinguish different types of hydrodynamic conditions I-III (Table 1): a symmetrical $M_{2}$ tide, without additional components, or with superimposed either a small residual current or an $M_{4}$ component.

[25] For later use in section 4.3, we define a fourth case IV corresponding to the access channel to the Port of Amsterdam. As reported by van de Kreeke et al. [2002], the tidal conditions are asymmetrical (see Table 1). For all cases I-IV, the parameter values for the BBF code are given in Appendix B.

[26] For these four settings, Table 1 shows the characteristics of the fgm (wavelength and e-folding time of the growth) along with the values of the dimensionless parameters used in the analytical solution. Comparing the cases IIII, we see that neither wavelength, nor growth rate is affected by the tidal asymmetry. This also applies to the real parts of $\lambda$ and $\mu$. However, as can be expected, disrupting the symmetry does lead to nonzero values of $\sigma$, $\sigma_{g}, \lambda_{i}$ and $\mu_{i}$. Migration is strongest when adding a residual current, rather than an $M_{4}$ component of similar strength, which can also be inferred from the results by Besio et al. [2003b].

[27] We furthermore notice that the values of the migration speed $\sigma$ and the group speed $\sigma_{g}$ hardly differ. This means that, according to linear theory and in the vicinity of the fgm, the sandwaves behave in a nondispersive way. More details on the parameter settings are given in Appendix B.

\subsection{Comparison Between Approximation and Numerical Solution}

[28] Now let us compare the analytical and numerical results, distinguishing between two-dimensional and threedimensional settings. In two dimensions (neglecting the transverse wave number $\ell$, see Appendix A), we find excellent agreement between the analytical and numerical results with respect to growth, expansion and migration (Figure 5). Other simulations not reported here confirm this finding, both for symmetric and asymmetric tidal conditions.

[29] In three dimensions the performance is also satisfactory (Figure 6). The analytical approximation accurately reproduces the migration and expansion of the packet, even including the slight curvature of the individual sandwave crests (which is related to $\mu_{i}$, see section 3.2). Other simulations, however, indicate that for smaller values of the initial topographic width $B$ the agreement is worse. This is because the quadratic approximation of the dispersion relationship appears to be somewhat crude. As shown by the examples in Table 2, some of the higher-order derivatives turn out to be significant. These terms are neglected in the quadratic approximation (3) of the dispersion relationship, 
(a) $\tau=0$

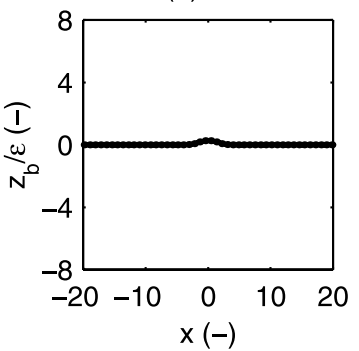

(b) $\tau=1$

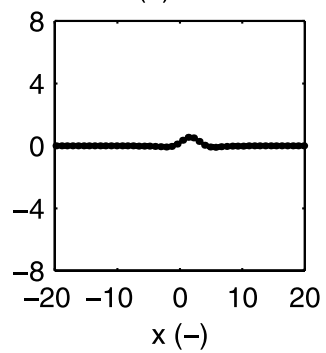

(c) $\tau=2$

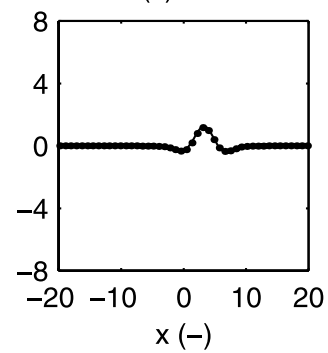

(d) $\tau=3$

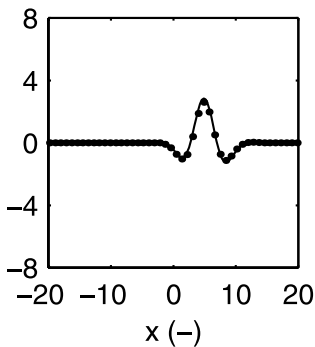

(e) $\tau=4$

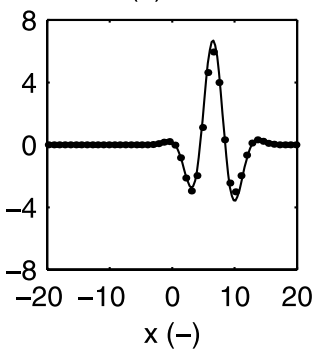

Figure 5. Comparison between approximation (solid lines) and numerical solution (dotted lines) for a disturbance with $L=2$ (side view). Tidal conditions are taken from case II in Table 1 (unit length is $1 / \hat{k}^{*}$ $\sim 68 \mathrm{~m}$; unit time is 10.8 years), with the residual current causing migration from left to right.

which affects the performance regarding lateral diffusion of the topography. This is most noticeable for small values of $B$.

\subsection{Application to Sand Extraction}

[30] For negative values of $\epsilon$, the initial topography (2) can be used to represent a sandpit or a navigation trench. For our approach to be applicable, the characteristic length should be of the order of the length scale of sandwaves $(100-1000 \mathrm{~m})$. These conditions are satisfied by the access channel to the Port of Amsterdam, known as the IJ channel. With a width of $600 \mathrm{~m}$, a maximum trench depth of $3 \mathrm{~m}$ at an average water depth of $17 \mathrm{~m}$ [van de Kreeke et al., 2002], it can indeed be seen as a relatively small disturbance of the seabed with an appropriate length scale. Furthermore, it should be noted that part of the access channel lies in an area with tidal sandwaves. The corresponding tidal conditions are given in Table 1 (case IV). For this typical case, we make a comparison between our model results and those by van de Kreeke et al. [2002], who used an alternative approach to describe this morphodynamic problem.

[31] First, we briefly describe the morphodynamic model by van de Kreeke et al. [2002], aimed at obtaining analytical solutions. To that end, they make three assumptions: (1) a depth-averaged flow model suffices, (2) the tidal flow, with components as in Table 1, is perpendicular to the main axis of the trench, and (3) the trench depth is small compared to the undisturbed water depth. As a result, the along-trench flow is neglected and the (depth-averaged) flow field is fully governed by continuity. A theoretical analysis of the linearized morphodynamic model then leads to a linear advection-diffusion equation for the seabed elevation. As a result, an initially Gaussian profile migrates in the direction of the residual sediment flux (northward, for the IJ Channel), increasing in width, while maintaining its Gaussian shape. van de Kreeke et al. [2002] estimate migration rates at $1.3 \mathrm{~m} \mathrm{yr}^{-1}$ for bed load transport, and (a) $\tau=0$ (app)

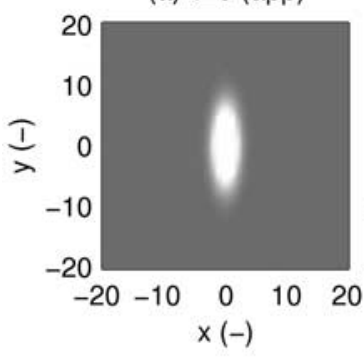

(f) $\tau=0$ (num)

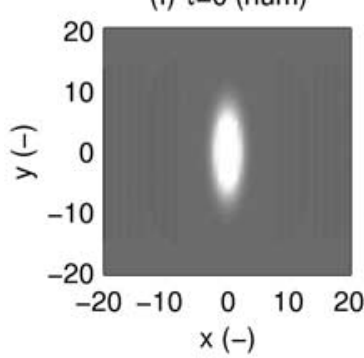

(b) $\tau=1$ (app)

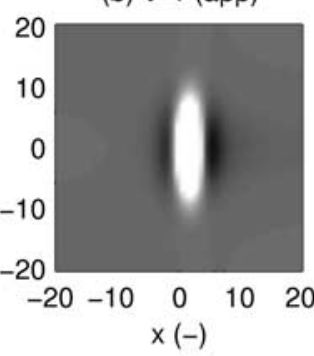

(g) $\tau=1$ (num)

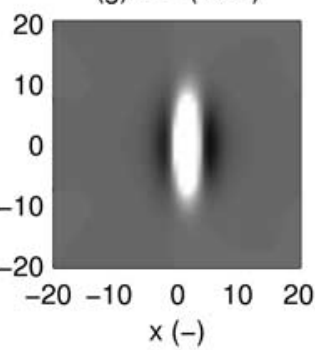

(c) $\tau=2$ (app)

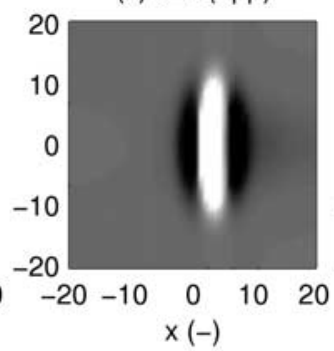

(h) $\tau=2$ (num)

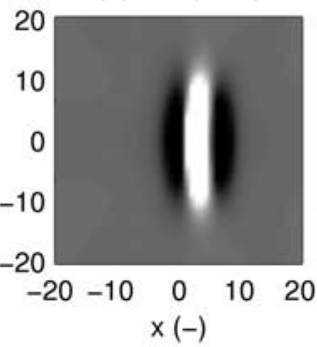

(d) $\tau=3($ app)

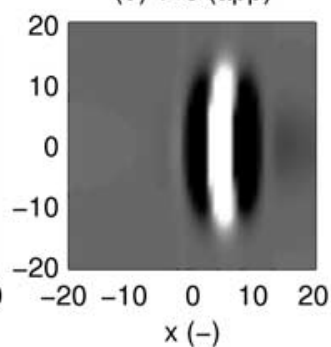

$x(-)$

(i) $\tau=3$ (num)

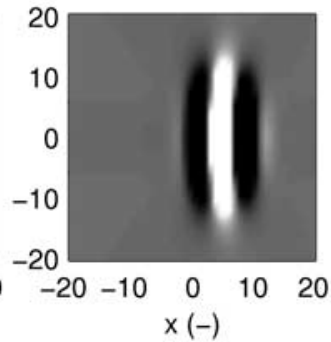

(e) $\tau=4$ (app)

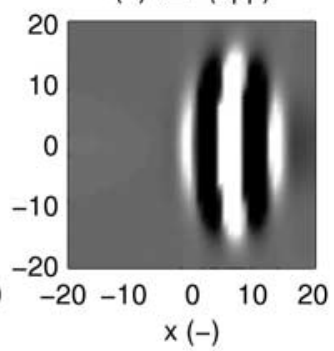

Figure 6. Comparison between (top) approximation and (bottom) numerical solution for an initial disturbance with $L=2$ and $B=6$ (top view). Tidal conditions are taken from case II in Table 1 (unit length is $1 / k^{*} \sim 68 \mathrm{~m}$; unit time is 10.8 years), with the residual current causing migration from left to right. 
Table 2. Higher-Order Derivatives of the Dispersion Relationship

\begin{tabular}{lcccc}
\hline & \multicolumn{4}{c}{ Dimensionless Value in fgm } \\
\cline { 2 - 5 } Derivative $^{\mathrm{a}}$ & $\mathrm{I}^{\mathrm{b}}$ & $\mathrm{II}^{\mathrm{b}}$ & $\mathrm{III}^{\mathrm{b}}$ & $\mathrm{IV}^{\mathrm{b}}$ \\
\hline$\frac{1}{6} \frac{\partial^{3} \omega}{\partial k^{3}}$ & 0.14 & $0.14+0.04 i$ & $0.14+0.01 i$ & $0.13+0.04 i$ \\
$\frac{1}{2} \frac{\partial^{3} \omega}{\partial k \partial \ell^{2}}$ & 0.88 & $0.88-0.49 i$ & $0.88-0.08 i$ & $0.86-0.48 i$ \\
$\frac{1}{24} \frac{\partial^{4} \omega}{\partial k^{4}}$ & -0.05 & $-0.05-0.01 i$ & $-0.05-0.001 i$ & $-0.05-0.01 i$ \\
$\frac{1}{4} \frac{\partial^{4} \omega}{\partial k^{2} \partial \ell^{2}}$ & -0.75 & $-0.75+0.47 i$ & $-0.75+0.01 i$ & $-0.70+0.46 i$ \\
$\frac{1}{24} \frac{\partial^{4} \omega}{\partial \ell^{4}}$ & 0.87 & $0.87-0.57 i$ & $0.87-0.09 i$ & $0.88-0.55 i$
\end{tabular}

${ }^{\mathrm{a}}$ By symmetry, terms containing a derivative to an odd degree in $\ell$ are zero.

${ }^{\mathrm{b}}$ Hydrodynamic conditions I-IV as given in Table 1 (also see section 4.1).

$1.8 \mathrm{~m} \mathrm{yr}^{-1}$ for suspended load transport. The initial rates of increase of the half-width are estimated at $0.33 \mathrm{~m} \mathrm{yr}^{-1}$ for bed load transport and $1.02 \mathrm{~m} \mathrm{yr}^{-1}$ for suspended load transport.

[32] Next, we use the analytical approximation (5) of our model to find estimates of the (initial) rates of pit migration, pit expansion and amplification/decay at the pit's center.

[33] 1. The migration speed of the pit can be estimated from the group velocity $\sigma_{g}$, as this is the speed at which (the envelope of) the sandwave packet migrates. In this example, we find a migration rate of about $10 \mathrm{~m} \mathrm{yr}^{-1}$.

[34] 2. Expansion of the packet is governed by the evolution of the ellipses, given by equations (10) and (11). Figure 7a shows the dependency of the initial rates of expansion on $L$ and $B$, which can be up to $\sim 10 \mathrm{~m} \mathrm{yr}^{-1}$.

[35] 3. Whether the elevation at the packet's center initially is amplified or decays, depends on the pit length $L$ and width $B$ as well as the parameters of the dispersion relationship (Figure 7b).

\subsection{Comparison With van de Kreeke et al. [2002]}

[36] The results from our model differ both qualitatively and quantitatively from those by van de Kreeke et al. [2002]. The qualitative implication of their assumption to employ a depth-averaged flow model is that details of the vertical flow structure are ignored. Therefore the dynamics of sandwaves, which is included in our model, is neglected by van de Kreeke et al. [2002], since this would require the description of vertical recirculation cells. A quantitative implication of including three-dimensional effects is a significant increase in migration and expansion rates. As reported by van de Kreeke et al. [2002], their predicted migration rate is somewhat smaller than that observed in the field, though of the right order of magnitude. The occurrence of higher migration rates in our model is mainly due to the vertical structure of the velocity profile which induces a higher bed shear stress on a wavy bed. Our analysis thus provides an alternative mechanism to explain the occurrence of higher migration rates.

[37] Also the assumption by van de Kreeke et al. [2002] of perpendicular flow has implications for the solution. The purely advective-diffusive character of their solution would be disturbed by the presence of an oblique rather than perpendicular tidal flow. Within a depth-averaged flow approach, such an obliqueness causes a deflection of the flow, the morphodynamic implication of which is the possible appearance of sandbanks [Roos and Hulscher, 2003]. This mechanism, relevant at length scales of 5$10 \mathrm{~km}$ and timescales of the order of decades to centuries, is not included in our model either.

[38] The qualitative and quantitative differences between our results and those by van de Kreeke et al. [2002] emphasize the importance of identifying the relevant morphodynamic mechanisms which control a particular phenomenon. In the present context, they are related to the potential of an area to develop tidal sandwaves.

\section{Discussion}

\subsection{Physical Mechanisms}

[39] The physical mechanisms behind the dynamics of a sandwave packet are similar to the physics of a spatially unconstrained sandwave pattern. However, they manifest themselves in a geometrically different way, which introduces new elements: a different amplitude evolution, an expanding envelope and the role of the group speed.

[40] The evolution at the packet's center differs from the purely exponential growth of the fastest growing mode in a spatially unconstrained pattern. This is seen from the possible initial decay of the amplification factor (as plotted in Figure 4), which shows that the wave spectrum of the packet consists of both growing and decaying modes. The relative presence of growing and decaying modes, depending on $L, B, \lambda$ and $\mu$, determines whether decay or growth initially dominates at the packet center. Obviously, the ultimate tendency of the packet to grow exponentially is due to the fastest growing mode eventually dominating the evolution of the spectrum.

[41] The evolution of the envelope $E_{L B}$, as given by equations (10) and (11), shows how the locally triggered instability gradually spreads over the domain. The longitudinal expansion of the sandwave packet can be directly understood from the growing amplitudes of the waves within the packet (see Figure 5): the growth of an individual crest automatically creates a trough on either side, and the further deepening of this trough similarly will create a new crest next to it. The lateral expansion of the packet, i.e., the

(a) packet expansion $(\tau=0)$

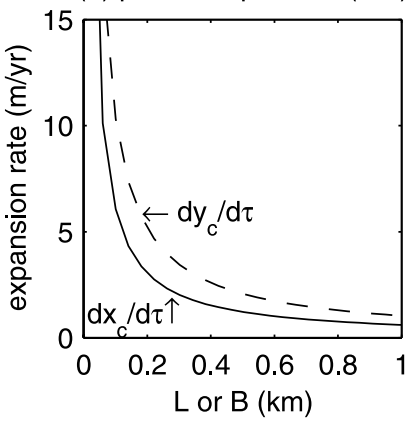

(b) evolution at center $(\tau=0)$

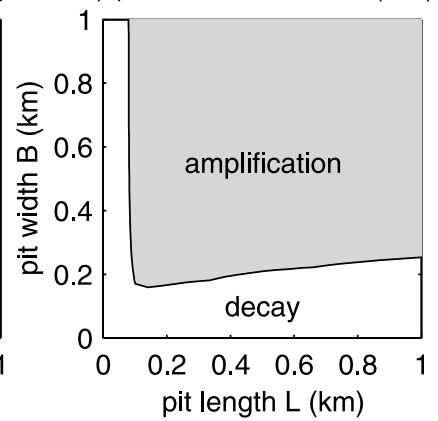

Figure 7. Initial evolution of a sandpit subject to the tidal conditions IV (Table 1): (a) rates of expansion in the longitudinal (solid line) and lateral (dashed line) direction and (b) amplification (shaded) or decay, at the pit's center, as a function of $L$ and $B$. 
elongation of the crests and troughs (as seen in plan view; see Figure 6), is driven by the downhill sediment flux.

[42] As can be seen from equations (5)-(11), the parameters $\lambda$ and $\mu$ influence both amplitude evolution and packet expansion. These complex-valued parameters describe the curvature of the growth rate $\omega$ in the fastest growing mode. As noted in section 2, the gravitational bed slope effect has a quadratic influence on $\omega_{r}$, and thus a diffusive effect on topography. The imaginary parts of $\lambda$ and $\mu$ reflect the curvature of $\omega_{i}$ and are therefore indirectly related to variations of the migration rate, given by $-\omega_{i}^{*} /\left(k^{* 2}+\right.$ $\left.\ell^{* 2}\right)^{1 / 2}$, around the fastest growing mode. This indirect relation with migration rate complicates the physical interpretation of $\lambda_{i}$ and $\mu_{i}$, which we do not pursue further.

[43] Finally, the focus on spatially constrained topographies has introduced a universal property of wave theory to our analysis of sandwave dynamics: the role of the group speed $\sigma_{g}$ besides that of the phase speed $\sigma$. As noted in section 4.1, our results indicate that $\sigma \approx \sigma_{g}$ in the fastest growing mode (Table 1).

\subsection{Assumptions and System Properties}

[44] Our approach, based on existing models of sandwave formation [Hulscher, 1996; Gerkema, 2000; Komarova and Hulscher, 2000; Besio et al., 2003a, 2003b], as such inherits the assumptions and restrictions of these approaches. These for example require tide-dominated conditions and sediment abundance. In addition, to warrant the validity of the linear approach, the amplitude of the bed elevation should be small with respect to the water depth (implying $\epsilon \ll 1$ ). However, from the nonlinear numerical sandwave evolution model of Németh et al. [2003], it can be inferred that the linear theory behaves well up to amplitudes of about $5-10 \%$ of the average water depth. This gives us confidence in the applicability of the linear approach, also in the finite amplitude regime. Despite the extended applicability of the linear theory, we emphasize a fundamental restriction of a linear approach: its inability to describe sandwaves in a finite amplitude morphodynamic equilibrium. The stability properties of such a finite amplitude equilibrium are therefore a different subject, beyond the scope of the present study.

[45] The sandwave packet analysis is based on the universal properties of existing models for sandwave formation: the presence of a fastest growing mode and the validity of a quadratic approximation of the dispersion relationship around the fgm. The sensitivity of these models with respect to the viscous and slip parameter has been extensively investigated for the model by Hulscher [1996], both regarding the type of pattern and the characteristics of the pattern (wavelength and timescale). For example, sandwaves occur for relatively large values of the slip parameter and moderate values of the viscous parameter. Using local estimates of these parameters, good agreement was obtained between predicted and observed sandwaves in the North sea [Hulscher and van den Brink, 2001]. For a discussion of the influence of the different tidal constituents on the migration rates, we refer to Besio et al. [2004].

\subsection{Local Geometry and Sandwave Modeling}

[46] The main advantage of our analysis is the local character of the topographic disturbance and the resulting sandwave packet. This contrasts with the existing linear theories, which describe patterns of infinite spatial extent. The present analysis can therefore be used as an analytical tool to validate more complex numerical models, especially with nonperiodic boundary conditions. This can be done in both a three-dimensional (Delft3d, Telemac) and a twodimensional setting (such as the nonlinear 2DV model by Németh et al. [2003]). The small-amplitude dynamics of a local topographic disturbance obtained with these models should match the analytical solution of our theory.

[47] Alternatively, heuristic sandwave models are currently being developed to describe the nonlinear plan view dynamics of finite-amplitude sandwave patterns [van den Berg and van Damme, 2004]. This approach combines linear growth characteristics (simplified or taken from existing process-based models) with a nonlinear, heuristic damping mechanism. Our theoretical analysis helps understanding how the linear part of such a model manifests itself in a three-dimensional geometry $z=z_{b}(x, y)$.

[48] Within the linear approach, Fourier modes do not interact and their evolution is simply superimposed. This implies that the results of different linear models, each valid at different spatial scales (for example, those of sandwaves and sandbanks), could also be superimposed. In this way, an impression of the small-amplitude behavior at different spatial scales can be easily obtained without the need for a unique model fully covering all relevant length scales by itself.

\section{Conclusions}

[49] We have studied the initial evolution of a local topographic disturbance subject to the morphodynamic mechanisms of sandwave formation. On the basis of the existing knowledge from linear models on sandwave formation, we have derived an analytical method to describe the seabed evolution. It is fast, insightful and captures the essential elements revealed by the alternative, more laborious and less transparent numerical solution. The results display a packet with a growing and migrating pattern of sandwaves, an expanding and migrating envelope, and even details like wavelength variations within the packet and the tendency toward a plan view curvature of the individual crests.

[50] Practical applications of this theory can, for example, be found in the context of sand extraction and channel dredging. Making the initial topography represent an offshore sandpit (or navigation trench), we investigated the sensitivity of the topographic evolution to its length and width. The results qualitatively and quantitatively differ from predictions made with a model, that ignores sandwave dynamics. The inclusion of sandwave dynamics introduces the instability associated with sandwave formation and leads to significantly higher rates of migration and expansion.

\section{Appendix A: Analysis in Two Dimensions}

[51] The two-dimensional counterparts of the bed evolution equation (1) and the initial topography (2) read

$$
\begin{gathered}
z_{b}(x, \tau)=\int_{0}^{\infty} \tilde{z}_{b}(k) e^{\omega(k) \tau} e^{i k x}+\text { c.c. } \\
z_{b}(x, 0)=\frac{\epsilon e^{-x^{2} / L^{2}}}{L \sqrt{\pi}}=\frac{\epsilon}{2 \pi} \int_{-\infty}^{\infty} e^{-\frac{1}{4} k^{2} L^{2}} e^{i k x} \mathrm{~d} k,
\end{gathered}
$$


respectively, such that $\int z_{b} \mathrm{~d} x=\epsilon$. Here $\omega(k)$ is the dispersion relationship as a function of the longitudinal wave number $k$ only. Its two-dimensional approximation is given by equation (3), with $\mu=0$. The analytical solution is identical to equation (5), with $A_{L B}$ and $E_{L B}$ replaced by

$$
\begin{gathered}
A_{L}(\tau)=\frac{e^{\left[1-\gamma_{L}(\tau)\right] \tau}}{\sqrt{\pi\left(L^{2}+4 \lambda \tau\right)}} \\
E_{L}(x, \tau)=\exp \left\{\frac{-\left[x-\sigma_{g} \tau\right]^{2}}{L^{2}+4 \lambda \tau}\right\},
\end{gathered}
$$

respectively. Finally, the two-dimensional counterpart of the numerical solution (12) neglects the summation over the transverse wave number $\ell$ :

$$
z_{b}^{\text {num }}(x, \tau)=\left[\Delta_{k} \sum_{j=1}^{N_{k}} \bar{Z}_{j} e^{\bar{\omega}_{j} \tau} e^{i \bar{k}_{j} x}\right]+\text { c.c. },
$$

where $Z_{j}=\tilde{z}_{b}\left(k_{j}\right), \bar{Z}_{j}=\frac{1}{2}\left(Z_{j-1}+Z_{j}\right)$ and $\bar{\omega}_{j}=\frac{1}{2}\left(\omega_{j-1}+\omega_{j}\right)$.

\section{Appendix B: Background of the BBF Code}

\section{B1. Introduction}

[52] The calculations in this study have been carried out using a numerical sandwave model, termed the BBF code. It is the three-dimensional extension of the linear stability analysis by Besio et al. [2003a], who determined the time development of a two-dimensional bottom waviness on an otherwise flat seabed, subject to a tidal flow.

\section{B2. Physics}

[53] The hydrodynamics is based on the Reynolds averaged momentum equations, where the Boussinesq assumption, along with a constant value of the eddy viscosity, is used to model the turbulent stresses. A constant eddy viscosity provides an acceptable description of the flow induced by tide propagation, provided the no-slip condition at the bottom is replaced by a partial slip condition [Engelund, 1974]. Here, a stress parameter $\tilde{s}$ appears, which should be properly chosen.

[54] Because the morphodynamic timescale (of the order of years) turns out to be much larger than the tidal timescale (12 hours $25 \mathrm{~min}$ ), the hydrodynamic problem can be decoupled from the morphodynamic one by neglecting the bottom changes $\partial z_{b} / \partial t$ in the hydrodynamic problem. Moreover, scaling arguments show that Coriolis terms and the local acceleration terms can be safely neglected. Indeed, the ratio between these terms and the other terms in the momentum equations turns out to be of order $1 / \hat{r}$, where the dimensionless parameter $\hat{r}$ (equal to the ratio of the tidal excursion amplitude and the mean water depth) is much larger than one.

[55] The morphodynamic module combines a sediment continuity equation with a sediment transport predictor. The latter is based on the bed load transport formula by MeyerPeter and Müller [1948], corrected to account for local bed slope effects. The dynamics of the sea bottom is forced by a unidirectional oscillatory tidal current aligned with the $x$ axis and consisting of an $M_{2}$ component, which is supposed to be the largest, an $M_{4}$ component and a residual current (see Table 1).

\section{B3. Solution Method}

[56] The bottom waviness, characterized by wave numbers $k^{*}$ and $\ell^{*}$ in the $x^{*}$ and $y^{*}$ direction, respectively, is assumed to have an amplitude $a^{*}$ that is small compared to the local water depth. Hence the solution can be expanded as a power series of the (infinitesimally) small parameter $a=$ $a^{*} / H^{*}$.

[57] At the leading order of approximation, $\mathcal{O}\left(a^{0}\right)$, the bottom is flat and the velocity profile of the tidal flow can be easily determined (see Besio et al. [2003a] for details). Moreover, the sediment transport predictor provides a spatially uniform sediment flux, so the bottom remains flat.

[58] At $\mathcal{O}(a)$, the flow field turns out to be proportional to $\exp \left[i\left(k^{*} x^{*}+\ell^{*} y^{*}\right)\right]$, through depth-dependent amplitudes which satisfy a system of coupled ordinary equations. To solve this numerically, a second-order Runge-Kutta scheme is used, along with a shooting procedure which allows boundary conditions to be imposed simultaneously at the bottom and at the free surface. Finally, the sediment continuity equation and the $\mathcal{O}(a)$ contributions to the sediment transport rates provide the complex-valued growth rate $\omega^{*}\left(k^{*}, \ell^{*}\right)$, as introduced in section 2 . Further details of the analysis can be found in the work of Besio et al. [2003a].

\section{B4. Dimensionless Parameters}

[59] The analysis is characterized by the following dimensionless parameters: particle mobility number $\psi_{d}$, particle Reynolds number $R_{p}$, ratio of tidal excursion and water depth $\hat{r}$, viscous parameter $\hat{\mu}$, slip parameter $\tilde{s}$ and bed slope coefficient $\gamma$. For a detailed description of these parameters, we refer to Besio et al. [2003a]. For the conditions I-III in Table 1, we adopt the following values: $\psi_{d}=2.42 \cdot 10^{-3}, R_{p}=11.4, \hat{r}=214, \hat{\mu}=250, \hat{s}=1.5, \gamma=$ 0.1 . For the IJ channel (case IV), for which van de Kreeke et al. [2002] use a mean water depth of $17 \mathrm{~m}$ and a grain size of $200 \mu \mathrm{m}$, appropriate values turn out to be: $\psi_{d}=$ $1.75 \cdot 10^{-3}, R_{p}=11.4, \hat{r}=231, \hat{\mu}=250, \hat{s}=1.5, \gamma=0.1$. An overview of the corresponding tidal constituents is given in Table 1.

[60] Acknowledgments. This research has been carried out within the EU project HUMOR (contract EVK3-CT-2000-00037). Two authors (P.B. and G.V.) acknowledge the EU project SANDPIT (contract EVK-CT-200100053). The authors thank Huib de Vriend for his comments.

\section{References}

Benjamin, T. B. (1961), The development of three-dimensional disturbances in an unstable film of liquid flowing down an inclined plane, J. Fluid Mech., 10, 401-419.

Besio, G., P. Blondeaux, and P. Frisina (2003a), A note on tidally generated sand waves, J. Fluid Mech., 485, 171-190.

Besio, G., P. Blondeaux, M. Brocchini, and G. Vittori (2003b), Migrating sand waves, Ocean Dyn., 53, 232-238, doi:10.1007/s10236-0030043-X.

Besio, G., P. Blondeaux, M. Brocchini, and G. Vittori (2004), On the modeling of sand wave migration, J. Geophys. Res., 109, C04018, doi:10.1029/2002JC001622

Engelund, F. (1974), Flow and bed topography in channel bends, J. Hydrol. Div. Am. Soc. Civ. Eng., 100, 1631-1648.

Gaster, M. (1968), The development of three-dimensional wave packets in a boundary layer, J. Fluid Mech., 32, 173-184.

Gaster, M. (1981), Propagation of linear wave packets in laminar boundary layers, AIAA J., 19, 419-423. 
Gaster, M. (1982), Estimates of the errors incurred in various asymptotic representations of wave packets, J. Fluid Mech., 121, 365-377.

Gaster, M., and A. Davey (2003), The development of three-dimensional wave packets in unbounded parallel flows, J. Fluid Mech., 32, 801-808.

Gerkema, T. (2000), A linear stability analysis of tidally generated sand waves, J. Fluid Mech., 417, 303-322.

Hulscher, S. J. M. H. (1996), Tidal-induced large-scale regular bed form patterns in a three-dimensional shallow water model, J. Geophys. Res., $101,20,727-20,744$.

Hulscher, S. J. M. H., and G. M. van den Brink (2001), Comparison between predicted and observed sand waves and sand banks in the North Sea, J. Geophys. Res., 106, 9327-9338.

Komarova, N. L., and S. J. M. H. Hulscher (2000), Linear instability mechanisms for sand wave formation, J. Fluid Mech., 413, 219-246.

Meyer-Peter, E., and R. Müller (1948), Formulas for bed load transport, in Proceedings of the 2nd Meeting of the International Association for Hydraulic Structures Research, pp. 39-64, Int. Assoc. for Hydraul. Res., Delft, Netherlands.

Morse, P. M., and H. Feshbach (1953), Methods of Theoretical Physics, vols. I-II, McGraw-Hill, New York.

Németh, A. A. (2003), Modelling offshore sand waves, Ph.D. Thesis, 140 pp., Univ. of Twente, Enschede, Netherlands.

Németh, A. A., S. J. M. H. Hulscher, and H. J. De Vriend (2002), Modelling sand wave migration in shallow shelf seas, Cont. Shelf. Res., 22, $2795-2806$
Németh, A. A., S. J. M. H. Hulscher, and H. J. De Vriend (2003), Offshore sand wave dynamics: Engineering problems and future solutions, Pipeline Gas J., 230, 67-69.

Roos, P. C., and S. J. M. H. Hulscher (2003), Large-scale seabed dynamics in offshore morphology: Modeling human intervention, Rev. Geophys., 41(2), 1010, doi:10.1029/2002RG000120.

Terwindt, J. H. J. (1971), Sand waves in the Southern Bight of the North Sea, Mar. Geol., 71, 51-67.

van den Berg, J., and R. M. J. van Damme (2004), A simplified sand wave model, paper presented at the 2nd International Workshop on Marine Sandwave and River Dune Dynamics (MARID), Univ. of Twente, Enschede, Netherlands.

van de Kreeke, J., S. E. Hoogewoning, and M. Verlaan (2002), An analytical model for the morphodynamics of a trench in the presence of tidal currents, Cont. Shelf Res., 22, 1811-1820.

P. Blondeaux and G. Vittori, Department of Environmental Engineering, University of Genova, Via Montallegro 1, I-16145 Genova, Italy. (blx@diam.unige.it; vittori@diam.unige.it)

S. J. M. H. Hulscher and P. C. Roos, Water Engineering and Management, Faculty of Engineering Technology, University of Twente, P.O. Box 217, 7500 AE Enschede, Netherlands. (s.j.m.h.hulscher@utwente. nl; p.c.roos@utwente.nl) 